24. Gamelin L. et al. Prevention of oxaliplatin-related neurotoxicity by calcium and magnesium infusions: a retrospective study of 161 patients receiving oxaliplatin combined with 5-Fluorouracil and leucovorin for advanced colorectal cancer. Clin Cancer Res. 2004;10(12 pt 1):4055-4061.

25. Grothey A, et al. Intravenous calcium and magnesium for oxaliplatin-induced sensory neurotoxicity in adjuvant colon cancer: NCCTG N04C7. J Clin Oncol. 2011;29(4):421-427.

26. Gamelin L, et al. Oxaliplatin-related neurotoxicity: interest of calcium-magnesium infusion and no impact on its efficacy. J Clin Oncol. 2008; 26(7):1188-1189.

27. Hochster HS, Grothey A, Childs BH. Use of calcium and magnesium salts to reduce oxaliplatin-related neurotoxicity. J Clin Oncol. 2007;25(25):4028-4029.

28. Loprinzi C, et al. Phase III randomized, placebo (PL)-controlled, double-blind study of intravenous calcium/magnesium (CaMg) to prevent oxaliplatin-induced sensory neurotoxicity (sNT), N08CB: An alliance for clinical trials in oncology study. J Clin Oncol. In press.

29. van der Hoop RG, et al Prevention of cisplatin neurotoxicity with an $\mathrm{ACTH}(4-9)$ analogue in patients with ovarian cancer. N Engl J Med. 1990;322(2):89-94.

30. Hovestadt A, van der Burg ME, Verbiest HB, van Putten WL, Vecht CJ. The course of neuropa- thy after cessation of cisplatin treatment, combined with Org 2766 or placebo. J Neurol. 1992; 239(3):143-146.

31. van Gerven JM, et al. The effects of an ACTH (4-9) analogue on development of cisplatin neuropathy in testicular cancer: a randomized trial. J Neurol. 1994;241(7):432-435.

32. van Kooten B, et al. A pilot study on the influence of a corticotropin (4-9) analogue on Vinca alkaloid-induced neuropathy. Arch Neurol. 1992; 49(10):1027-1031.

33. Koeppen S, et al. Lack of neuroprotection by an ACTH (4-9) analogue. A randomized trial in patients treated with vincristine for Hodgkin's or non-Hodgkin's lymphoma. J Cancer Res Clin Oncol. 2004;130(3):153-160.

34. Roberts JA, Jenison EL, Kim K, Clarke-Pearson D, Langleben A. A randomized, multicenter, double-blind, placebo-controlled, dose-finding study of ORG 2766 in the prevention or delay of cisplatin-induced neuropathies in women with ovarian cancer. Gynecol Oncol. 1997;67(2):172-177.

35. Cascinu S. et al. Neuroprotective effect of reduced glutathione on oxaliplatin-based chemotherapy in advanced colorectal cancer: a randomized, double-blind, placebo-controlled trial. J Clin Oncol. 2002;20(16):3478-3483.

36. Milla P, Airoldi M, Weber G, Drescher A, Jaehde U,
Cattel L. Administration of reduced glutathione in FOLFOX4 adjuvant treatment for colorectal cancer: effect on oxaliplatin pharmacokinetics, Pt-DNA adduct formation, and neurotoxicity. Anticancer Drugs. 2009;20(5):396-402.

37. Smyth JF, et al. Glutathione reduces the toxicity and improves quality of life of women diagnosed with ovarian cancer treated with cisplatin: results of a double-blind, randomised trial. Ann Oncol. 1997; 8(6):569-573

38. Bianchi, G. et al. Symptomatic and neurophysiological responses of paclitaxel- or cisplatin-induced neuropathy to oral acetyl-L-carnitine. EurJ Cancer. 2005; 41(12):1746-1750.

39. Hershman DL, et al. Randomized double-blind placebo-controlled trial of acetyl-L-carnitine for the prevention of taxane-induced neuropathy in women undergoing adjuvant breast cancer therapy. J Clin Oncol. 2013;31(20)2627-2633.

40. André T, et al. The IDEA (International Duration Evaluation of Adjuvant Chemotherapy) Collaboration: Prospective Combined Analysis of Phase III Trials Investigating Duration of Adjuvant Therapy with the FOLFOX (FOLFOX4 or Modified FOLFOX6) or XELOX (3 versus 6 months) Regimen for Patients with Stage III Colon Cancer: Trial Design and Current Status. Curr Colorectal Cancer Rep. 2013; 9:261-269.

\title{
Hostile takeover: fungal protein promotes host cell invasion
}

\author{
J. Andrew Alspaugh
}

Department of Medicine, Department of Molecular Genetics and Microbiology, Duke University School of Medicine, Durham, North Carolina, USA.

\begin{abstract}
The ability to suppress the immune system has lead to great advances in transplant technology and treatment of autoimmune diseases. Unfortunately, the immunosuppression of these patients has led to the rise of opportunistic infections by organisms that are recalcitrant to current prophylactic strategies. One such example is the increase of mucormycosis, an invasive infection caused by filamentous fungi of the order Mucorales. In this issue of the JCI, Gebremariam and colleagues determined that spore coat homolog $(\mathrm{CotH})$ proteins are required for angioinvasion and that these proteins are unique to Mucorales. Their findings provide a potential therapeutic target for prevention and treatment of mucormycosis.
\end{abstract}

\section{The rise of mucormycosis}

Recent medical advances have made remarkable progress in treating previously refractory conditions. More aggressive and targeted cancer chemotherapies have vastly improved outcomes for many malignancies. Inhibition of TNF- $\alpha$ activity now affords better control of various autoimmune disorders. Moreover, advances in solid organ transplantation have dramatically improved the lives of many patients

Conflict of interest: The author has declared that no conflict of interest exists.

Citation for this article: J Clin Invest. 2014; 124(1):74-76. doi:10.1172/JCI73585. with organ failure syndromes. However, these revolutionary therapies considerably impair patient immunity.

Because of the increased infection risk in patients with highly immunocompromised states, clinicians have adopted concrete strategies for infectious disease prevention in many of these patient populations. Unfortunately, as our ability to suppress infections by the most common microbial pathogens has improved, other, less well-characterized infectious agents have begun to fill this clinical void. One very important example of this phenomenon is the increasing incidence of mucormycosis, an invasive infection caused by the Mucorales order of filamentous fungi $(1,2)$. Human pathogens in this fungal group include Rhizopus, Mucor, and Cunninghamella species.

The increased incidence of mucormycosis has been attributed to many factors, including the fact that Mucorales are much less susceptible to current antifungal agents than other fungal pathogens. Therefore, the use of standard antifungal drugs in prophylactic strategies is unlikely to successfully prevent this type of infection $(3,4)$. Mucormycosis occurs in association with a wide range of disorders. In addition to classical immunocompromised states, such as prolonged neutropenia and organ transplantation, conditions such as diabetic ketoacidosis (DKA), undernutrition, and iron chelation therapy also predispose patients to mucormycosis (2). Once established, invasive infections due to Mucorales frequently take an aggressive clinical course characterized by rapid tissue destruction. These infections are difficult to treat, requiring high-dose antifungal therapy and surgical debridement. Mortality in mucormycosis remains high, despite 


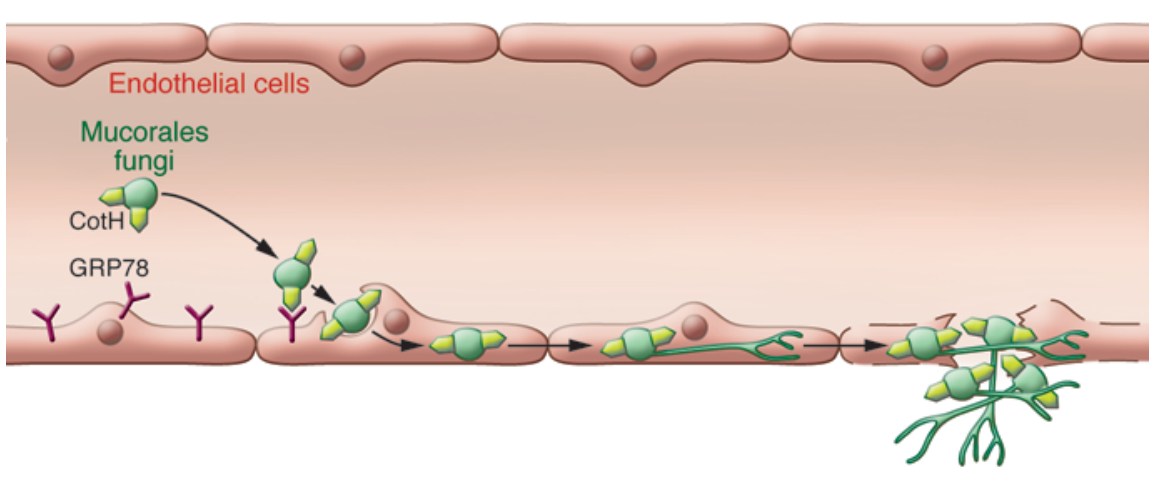

Figure 1

CotH proteins on the fungal surface promote angioinvasion. CotH proteins on the surface of Mucorales specifically bind to GRP78 on the surface of host endothelial cells. This interaction facilitates fungal invasion of the cell. Damage to the endothelial cells promotes angioinvasion and dissemination.

combined medical/surgical therapy $(5,6)$. Therefore, new insights into the pathogenesis of this fungal infection are needed in order to envision innovative strategies for prevention and treatment.

\section{Focus on angioinvasion}

The study in this issue by Gebremariam et al. (7) began with a classical clinical observation about mucormycosis that was used to infer unique aspects regarding the establishment and propagation of this disease. This infection tends to display angioinvasion, or progression along the micro- and macrovasculature (8). Clinically, angioinvasion results in tissue infarction, which complicates the infection and explains many of the principal manifestations of this disease. The authors decided to pursue the angioinvasive behavior of Mucorales to determine whether this fungal feature could offer insight into approaches to altering disease procession. Thus, they sought to determine whether specific molecular interactions exist between the fungal cell surface and the mammalian endothelium. Historically, there has been some debate among clinicians and pathologists as to whether fungal angioinvasion is the result of a specific interaction between host and fungus, or whether this process simply involves microbial growth along the path of least resistance within the infected tissue. The data now presented by Gebremariam et al. (7) strongly argue that the interaction of Rhizopus species and endothelial cells involves targeted molecular recognition between fungal and host cells, promoting microbial invasion and disease progression.

The authors previously identified glucose-regulated protein 78 (GRP78) as a component of mammalian cells that bound Rhizopus oryzae hyphae (9). GRP78 colocalized with the fungal hyphae during invasion of endothelial cells. Additionally, anti-GRP78 antibodies effectively prevented the adherence and endocytosis of $R$. oryzae, but not other fungal pathogens. Expression of GRP78 was induced in mice during DKA and in cultured epithelia exposed to excess glucose and iron, conditions that favor mucormycosis. Blocking this putative endothelial cell receptor with antibodies protected DKA mice from experimental mucormycosis (9).

\section{Mucorales-specific ligand promotes invasion}

In the present study, the authors identified fungal ligands that bind GRP78 (7). The data presented here compellingly argue that bacterial spore coat protein homolog $(\mathrm{CotH})$ proteins present on the $R$. oryzae cell surface mediate a specific interaction with GRP78, promoting fungal adherence and invasion (Figure 1). Heterologous expression of the R. oryzae CotH proteins resulted in mammalian cell invasion by the typically nonpathogenic yeast Saccharomyces cerevisiae. Additionally, interruption of $\mathrm{CotH}$ function in R. oryzae disrupted its invasive potential. Interference of the CotH/GRP78 interaction with anti-CotH antibodies resulted in a protective effect in a murine DKA model of mucormycosis. Interestingly, these $\mathrm{CotH}$ proteins were present on the surface of Mucorales, but do not appear to be produced by other fungal pathogens (7). This may explain the more extensive degree of angioinvasion observed in mucormycosis compared with other invasive fungal infections.

\section{Perspectives and future directions}

Many questions naturally arise when considering the results of these investigations. Mucorales are not adapted human pathogens, but rather environmental molds that infect patients with specific defects in immunity; therefore, the native role of $\mathrm{CotH}$ proteins remains to be determined. Do these fungal proteins promote interaction with potential food sources in the environment? Alternatively, do these fungal surface proteins provide some sort of protection against natural predators?

In addition to the important questions in fungal biology raised by these findings, there are equally important clinical implications. The specificity of the CotH proteins to Mucorales suggests that they could be effective therapeutic targets for preventing and treating mucormycosis. Would therapies directed against fungal angioinvasion be effective once these infections are well established in tissues? It would also be interesting to study whether disruption of fungal adherence and invasion could be used in various prevention strategies, in addition to targeted therapies of active Mucorales infections.

These studies also highlight the larger context of recent investigations into the interaction of the fungal cell surface with the infected host. Fungal surface molecules that act as adhesins, invasins, and immunogens are being increasingly characterized in diverse human pathogens. Vaccine strategies against various fungal surface epitopes have been explored, and clinical trials of resulting fungal vaccines are now being pursued (10). Additionally, the fungal cell surface often determines the nature and degree of immune activation during invasive fungal infections. Exposure of highly immunogenic fungal surface components may be beneficial in some settings, allowing infected hosts to recognize and rapidly clear invading microorganisms. Conversely, excessive immune activation could potentially accelerate host damage, which would result in allergic responses for superficial fungal conditions and worsen morbidity in invasive mycoses (11-13). Therefore, continued investigations are warranted into this important intersection of microbiology and host immune response. 
Address correspondence to: J. Andrew Alspaugh, Duke University Medical Center, Division of Infectious Diseases and International Health, 1543 Busse Building, Department of Medicine, Durham, North Carolina 27710, USA. Phone: 919.684.0045; Fax: 919.684.8902; E-mail: andrew.alspaugh@duke.edu.

1. Saegeman V, Maertens J, Meersseman W, Spriet I, Verbeken E, Lagrou K. Increasing incidence of mucormycosis in University Hospital, Belgium. Emerg Infect Dis. 2010;16(9):1456-1458.

2. Bitar D, et al. Increasing incidence of zygomycosis (mucormycosis), France, 1997-2006. Emerg Infect Dis. 2009;15(9):1395-1401.

3. Marty FM, Cosimi LA, Baden LR. Breakthrough zygomycosis after voriconazole treatment in recipients of hematopoietic stem-cell transplants. N Engl J Med. 2004;350(9):950-952.

4. Kontoyiannis DP, et al. Zygomycosis in a tertiary-care cancer center in the era of Aspergillus-active antifungal therapy: a case-control observational study of 27 recent cases. J Infect Dis. 2005; 191(8):1350-1360

5. Alspaugh JA, Perfect JR. Infections due to zygomycetes and other rare fungal opportunists. Semin Respir Crit Care Med. 1997;18(3):265-279.

6. Roden MM, et al. Epidemiology and outcome of zygomycosis: a review of 929 reported cases. Clin Infect Dis. 2005;41(5):634-653.

7. Gebremariam T, et al. CotH3 mediates fungal invasion of host cells during mucormycosis. J Clin Invest. 2014;124(1):237-250.

8. Frater JL, Hall GS, Procop GW. Histologic features of zygomycosis: emphasis on perineural invasion and fungal morphology. Arch Pathol Lab Med.
2001;125(3):375-378

9. Liu M, et al. The endothelial cell receptor GRP78 is required for mucormycosis pathogenesis in diabetic mice. J Clin Invest. 2010;120(6):1914-1924.

10. Schmidt CS, et al. NDV-3, a recombinant alum-adjuvanted vaccine for Candida and Staphylococcus aureus, is safe and immunogenic in healthy adults. Vaccine. 2012;30(52):7594-7600.

11. Crameri R, Blaser K. Allergy and immunity to fungal infections and colonization. Eur Respir J. 2002; 19(1):151-157.

12. Gravelat FN, et al. Aspergillus galactosaminogalactan mediates adherence to host constituents and conceals hyphal beta-glucan from the immune system. PLoS Pathog. 2013;9(8):e1003575.

13. O'Meara TR, Holmer SM, Selvig K, Dietrich F, Alspaugh JA. Cryptococcus neoformans Rim 101 is associated with cell wall remodeling and evasion of the host immune responses. MBio. 2013; 4(1):e00522-12.

\title{
More than skin deep: connecting melanocyte pigmentation and angiogenic diseases
}

\author{
Christopher D. Kontos
}

\author{
Department of Medicine, Division of Cardiology, Department of Pharmacology and Cancer Biology, \\ Duke University Medical Center, Durham, North Carolina, USA.
}

\begin{abstract}
Epidemiological studies have identified racial differences in susceptibility to numerous diseases, including several ocular and skin diseases characterized by increased vascular growth. In most cases, the specific mechanisms and genetic variants responsible for these differences have remained elusive. In this issue of the JCI, Adini et al. explore a direct connection between skin pigmentation and susceptibility to angiogenic diseases and identify an extracellular matrix protein that is regulated by melanogenesis and potently modulates angiogenesis.
\end{abstract}

\section{Skin pigmentation and angiogenesis dysfunction}

An important and ongoing challenge in the postgenomic era is the successful identification of gene variants that render certain individuals susceptible to specific diseases. For example, it is well known that individuals with less skin pigmentation have a higher incidence of angiogenic ocular and skin disease compared with darker-skinned individuals. Genetic variation drives differences in pigmentation; however, a genetic link between skin color and angiogenic disease has not been identified. Studies on ocular diseases indicate that individuals of European descent are twice as likely to develop age-related macular degeneration (AMD) as African-Ameri-

Conflict of interest: The author has declared that no conflict of interest exists.

Citation for this article: J Clin Invest. 2014;

124(1):76-79. doi:10.1172/JCI73559. cans or Asian-Americans (1). Furthermore, ocular melanoma is almost 20 times more common in light-skinned individuals (2), and several skin diseases, including melanoma and hemangioma, are more common in those of European descent $(3,4)$. The fact that these diseases are all characterized by increased vascular growth suggests a potential unexplored link between skin pigmentation and angiogenesis.

In this issue of the JCI, Adini and colleagues investigate the connection between skin color and disease susceptibility (5). The authors hypothesized that pigmented cells from light- or darkskinned individuals differentially express a proangiogenic factor. They observed that angiogenesis in the cornea and in healing wounds is considerably increased in C57-albino mice, which have decreased melanin synthesis due to a mutation in the tyrosinase gene, compared with their pigmented counterparts (C57BL/6 mice).
Using a cell-culture model of sprout formation, Adini et al. performed a detailed assessment of the effects of melanocyte-conditioned media on EC migration. Conditioned media were prepared by culturing melanocytes from albino and pigmented mice and, in a complementary fashion, from European- and African-Americans. The authors demonstrated that nonpigmented melanocytes secrete a factor that promotes microvascular EC migration and proliferation in vitro. These data are consistent with a melanocyte-secreted factor playing a role in promoting blood vessel growth.

\section{Fibromodulin: a potent angiogenic factor}

In order to identify the specific factor or factors responsible for the observed effects, the authors performed a targeted microarray that compared expression of genes predicted to encode secreted proteins in pigmented and nonpigmented melanocytes (5). Fibromodulin (FMOD), an ECM protein of the small leucine-rich proteoglycan (SLRP) family, was expressed to a much greater degree in nonpigmented melanocytes both in vitro and in vivo. Silencing or antibody blocking of FMOD substantially inhibited the angiogenic effects of conditioned media from nonpigmented mela- 